\title{
Digital/Analog Arithmetic with Continuous-Valued Residues
}

\author{
Behrooz Parhami \\ Department of Electrical and Computer Engineering \\ University of California, Santa Barbara, USA \\ parhami@ece.ucsb.edu
}

\begin{abstract}
Residue number system (RNS) representations, which allow fast addition and multiplication, have found niche applications in signal processing. These representations are based on integer-valued residue "digits" with respect to integer moduli. We introduce RNS representations with continuous or analog digits, and study their dynamic range, accuracy, and optimal choice of the moduli. Like positional number systems with continuous digits, our representations offer advantages in robustness and fault tolerance. As an interesting application, we point to recent findings in computational neuroscience that attribute a rat's uncanny ability to return to a home position, even in the absence of visual clues, to a related hex-grid-based residue representation of its position.
\end{abstract}

Keywords - Continuous-valued digits; Digital arithmetic; Hybrid analog/digital encoding; Number representation.

\section{Introduction}

Residue number system (RNS) representations have been studied extensively since the early days of digital computers [1-4]. Early high hopes for superfast arithmetic due to independent parallel operations on the residues were shattered by the realization that certain difficult arithmetic operations and decision processes can nullify much or all of this gain. Thus, practical applications remained limited to signal processing tasks dominated by addition and multiplication operations. An extensive body of RNS-related methods was already in place by 1986 [5]. More recently, the potential of RNS for low-power arithmetic has gained attention (e.g., [6]).

RNS is used to represent integers or scaled fixed-point values. Given pairwise relatively prime integer moduli $m_{k-1}>\cdots>m_{1}>m_{0}$, an integer $R$ is represented by its ordered set of residues $\left(r_{k-1}, \ldots, r_{1}, r_{0}\right)$ with respect to the $k$ moduli. The dynamic range of this number system, that is, the number of distinct uniquely represented integers, is $M$ $=m_{k-1} \cdots m_{1} m_{0}$. This range can be used for unsigned numbers $[0, M-1]$, signed numbers $[-\lfloor M / 2\rfloor,\lceil M / 2\rceil-1]$, or any other set of $M$ consecutive integers.

Addition, subtraction, and multiplication operations are performed independently on each residue. Given the RNS operands $S=\left(s_{k-1}, \ldots, s_{1}, s_{0}\right)$ and $T=\left(t_{k-1}, \ldots, t_{1}, t_{0}\right)$, their sum/difference and product are formed as follows (notationally, $|z|_{m}$ stands for $z \bmod m$ ):

$$
\begin{aligned}
& S \pm T \equiv\left(\left|s_{k-1} \pm t_{k-1}\right| m_{k-1}, \ldots,\left|s_{1} \pm t_{1}\right| m_{1},\left|s_{0} \pm t_{0}\right|_{m_{0}}\right) \\
& S \times T \equiv\left(\left|s_{k-1} \times t_{k-1}\right| m_{k-1}, \ldots,\left|s_{1} \times t_{1}\right| m_{1},\left|s_{0} \times t_{0}\right| m_{0}\right)
\end{aligned}
$$

Problems to be addressed in practical applications include choice of the moduli, forward/reverse conversions from/to binary format, and consideration of when/how to scale the intermediate results to avoid overflow. In what follows, we focus only on the dynamic range and its properties, in preparation for our discussion of the continuous-digit case. For details of RNS arithmetic and applications, the reader is referred to [3].

The example RNS in Fig. 1, with $m_{1}=4$ and $m_{0}=3$, has a dynamic range of $M=12$, which can be used to represent the natural numbers in $[0,11]$. The next natural number, 12 , is indistinguishable from 0 , so 11 is as high as we are allowed go. Now, forgetting for the moment that the residues are restricted to natural numbers, let us ponder the effect of having real-valued $r_{1}$ and $r_{0}$, represented, for example, by analog signals. If we change $\left(r_{1}, r_{0}\right)=(1.0,1.0)$ to $\left(r_{1}^{\prime}, r_{0}^{\prime}\right)=\left(1.0+\varepsilon_{1}, 1.0+\varepsilon_{0}\right)$, where $\varepsilon_{1}$ and $\varepsilon_{0}$ are absolute errors, we can still correctly decode the number, as long as both $\varepsilon_{1}$ and $\varepsilon_{0}$ are less than 0.5 .

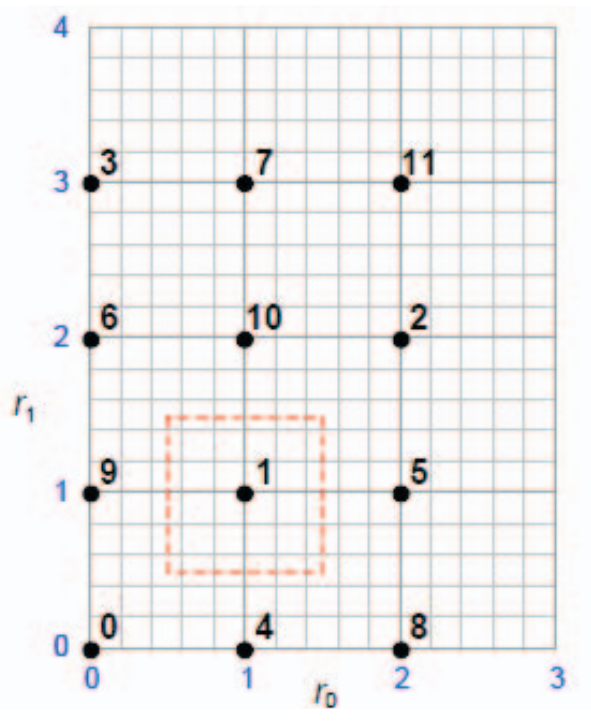

Fig. 1. Conventional RNS with the moduli 3 and 4. 
The horizontal and vertical separation of codes in Fig. 1 by a minimum of 1 unit allows us to correct any residue error of less than 0.5 , that is, $r_{1}^{\prime}$ and $r_{0}^{\prime}$ can vary anywhere inside the dashed box surrounding point 1 in Fig. 1, without causing a decoding error. If we need detection only, any error with a magnitude less than 1 is detectable. Similar error detection and correction capabilities may be provided by additional or redundant moduli, which lead to the extension of dynamic range, without actually utilizing all the values that would be representable [7].

Henceforth, we focus on two-residue number systems. With regard to dynamic range and other properties of interest, multiresidue systems can be handled by cascaded application of two-residue results. For example, the RNS that results from adding a third modulus $m_{2}=5$ to the RNS of Fig. 1 might be viewed and analyzed as one of the two-modulus systems $\{3,20\},\{4,15\}$, or $\{5,12\}$. All proofs are omitted in this paper.

\section{RNS with Continuous Residues}

The RNS of Fig. 1, viewed as having continuous or analog digits in $[0.0,3.0)$ and $[0.0,4.0)$, can be thought of as representing real numbers in $[0.0, \mu)$, where $\mu$ is the new dynamic range. But what would be an appropriate value for $\mu$ ? For ease of reference, we tackle this question in Example 1 below. Notationally, we switch to using $\mu$ for dynamic range and $\rho$ for residues, in lieu of $m$ and $r$, to signify that they are real-valued rather than integers.

Example 1: If, in Fig. 1, we drew lines with slope 1 to connect the values $0-11$ in ascending order, intermediate points on the lines would correspond to representations of nonintegers; e.g., the value 6.3 yields $\left(\rho_{1}, \rho_{0}\right)=(0.3,2.3)$, a point on the sloped line between 6 and 7. It is readily seen that we can go beyond 11 . With exact real-valued residues, the representable range will be $[0.0,12.0)$; otherwise, if $\varepsilon_{\max }$ denotes the maximum error in $\rho_{1}$ or $\rho_{0}$, that is, $\varepsilon_{\max }=\max \left(\left(\max \varepsilon_{1}\right),\left(\max \varepsilon_{0}\right)\right)$, then the range that ensures correct decoding within $\varepsilon_{\max }$ is $\left[0.0,12.0-\varepsilon_{\max }\right)$. We see that the dynamic range is on the order of $m_{1} m_{0}$. Note that even though the residues $\rho_{1}$ and $\rho_{0}$ are realvalued, relative primality of $m_{1}$ and $m_{0}$ remains a precondition for maximal range. More importantly, the dynamic range cannot be extended beyond $m_{1} m_{0}$, even if the real-valued residues are very accurate. This is because the wraparounds occur at precisely the same points for both real-valued and integer residues.

Consider the following analysis for solving the limited dynamic range problem. Given the RNS encoding of a value $R$, with the two residues containing (signed) errors of $\varepsilon_{1}$ and $\varepsilon_{0}$, the erroneous residues specify a point $R^{\prime}$ on the residue plane (Fig. 2). To decode this number, we might attempt to find the closest point $R^{\prime \prime}$ to it on one of the sloped lines of Fig. 1.

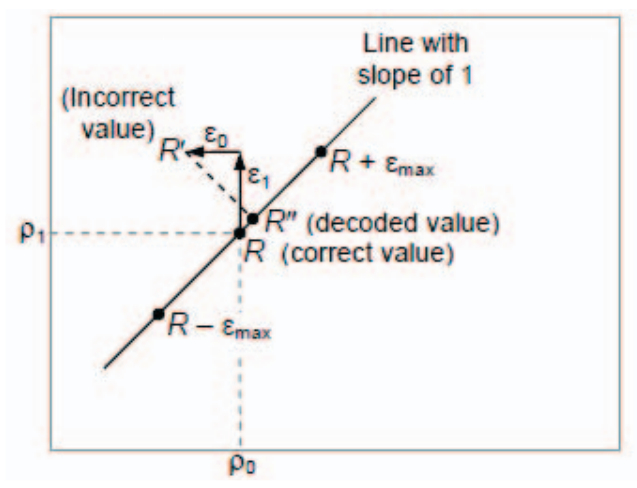

Fig. 2. Decoding error with continuous-digit RNS.

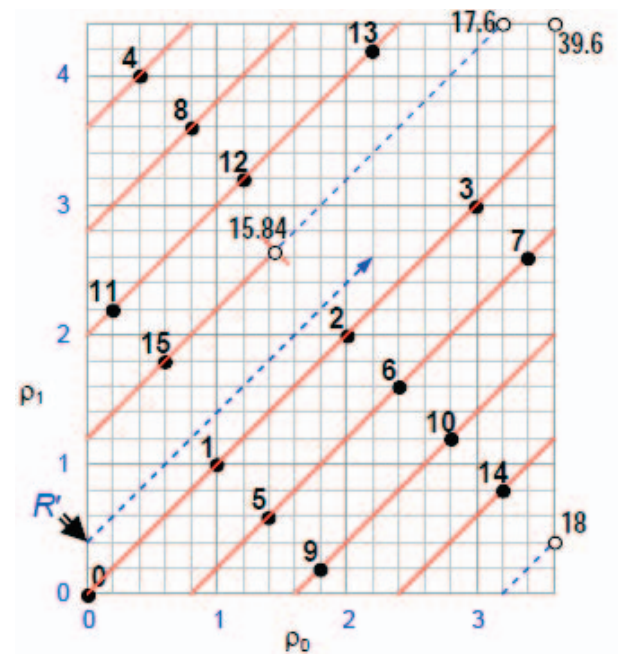

Fig. 3. Continuous-digit RNS with the moduli $\{3.6,4.4\}$.

For $\varepsilon_{1}, \varepsilon_{0}<0.5$, the closest line to $R^{\prime}$ is the same line that contains $R$ and the error in the decoded value $R^{\prime \prime}$ is at most $\varepsilon_{\max }$. This is because the coordinates of $R^{\prime \prime}$ in Fig. 2 are $\rho^{\prime \prime}{ }_{1}=\rho_{1}+\left(\varepsilon_{1}+\varepsilon_{0}\right) / 2$ and $\rho^{\prime \prime}{ }_{0}=\rho_{0}+\left(\varepsilon_{1}+\varepsilon_{0}\right) / 2$, leading to $R-\varepsilon_{\max } \leq R^{\prime \prime} \leq R+\varepsilon_{\max }$.

Now that we have introduced continuous residues or "digits," there is no compelling reason for insisting that the moduli $m_{1}$ and $m_{0}$ in a continuous-digit residue number system (CD-RNS) be whole numbers.

Example 2: Consider the CD-RNS with $\mu_{1}=4.4$ and $\mu_{0}=3.6$ (Fig. 3). The set $[0, \mu)$ of values represented fall on lines of slope 1 , with $\mu$ to be determined. The product $15.84=4.4 \times 3.6$ of the moduli would be a reasonable first guess. But we can go past 15.84, up to 18.0 (see the lower right corner of Fig. 3), without the horizontal or vertical line separations becoming less than 0.8 . Actually, to have an error of \pm 0.4 in a residue correctable, we must stop just under 17.6, associated with $\rho_{1}=0$ and $\rho_{0}=3.2$. This is because an error of +0.4 in both residues at $R=$ 17.6 will yield $(0.4,0.0)$ as the residue representation, which defines point $R^{\prime}$, thus causing the value 17.6 to be interpreted as $R^{\prime \prime}=0.2$ after correction according to the process depicted in Fig. 2. 
Example 2 shows that the same error tolerance applies to the extended range $[0.0,17.6)$, in lieu of $[0.0,15.84)$. That is, reducing the line separations from 1.0 in Fig. 1 to 0.8 in Fig. 3, a reduction factor of 1.25 , has allowed us to extend the dynamic range by about $11 \%$. Intuitively, the dynamic range not increasing by a factor of 1.25 is due to the waste from uneven line spacings in Fig. 3.

Example 3: If, in the CD-RNS of Example 2, we decide to go beyond 18.0 , the continuation of the dashed line from point $R^{\prime}$ of Fig. 3 suggests that we can do so, provided the error margin is lowered from 0.4 to 0.2 . In fact, we can go all the way to 39.6 , represented by the point at the upper right corner of Fig. 3, after wrapping around at 21.6, 22.0, 25.2, 26.4, 28.8, 30.8, 32.4, 35.2, and 36.0, successively. Again, to ensure that an error of \pm 0.2 in either residue is correctable, we have to end the dynamic range just under 39.4

Before continuing with our discussion of CD-RNS, it is worth noting that hybrid digital/analog arithmetic has been proposed in connection with positional number systems [8-10]. These representations are of interest due to their robustness, immunity to noise, simple carry-free analog operations, and energy economy. These positive attributes apply to our representations as well. The effects of errors in RNS have also been studied in the context of reverse conversion to binary $[11,12]$.

\section{Dynamic Range and Accuracy}

We continue with the assumption of an RNS with two moduli, $\mu_{1}>\mu_{0}$, and further assume, at the outset, that residue accuracy is dependent on the moduli, as defined below. We will subsequently consider the effect of accuracy as an independent parameter.

Theorem 1: Let $\sigma_{1}=\left|\mu_{1}\right|_{\mu_{0}}$. If the residues $\rho_{1}$ and $\rho_{0}$ are accurate to within $\pm \varepsilon_{\max }= \pm \sigma_{1} / 2$, then, the dynamic range of a CD-RNS with $\mu_{1}>\mu_{0}$ is $\mu=\mu_{0}\left(1+\left\lfloor\mu_{1} / \mu_{0}\right\rfloor\left\lfloor\mu_{0} / \sigma_{1}\right\rfloor\right)$.

Note that in Example 1, we derived the dynamic range as $\left[0.0,12.0-\varepsilon_{\max }\right)$. Similarly, in Examples 2 and 3, we subtracted $\varepsilon_{\max }$ from the upper end of a computed range to ensure that the last number included within the range is distinguishable from very small values at the beginning. In Theorem 1 and henceforth we omit the tiny $-\varepsilon_{\max }$ adjustment to simplify the expressions involved.

When $\mu_{1}$ is not close to an integral multiple of $\mu_{0}$, Theorem 1 essentially establishes a lower bound on the supportable dynamic range, given that its accuracy requirement for the residues is quite modest.

Example 4: If $\sigma_{1}=1.0$, then, provided residues are accurate to within \pm 0.5 , the dynamic range of Theorem 1 extends to $\mu=\mu_{0}\left(1+\left\lfloor\mu_{1} / \mu_{0}\right\rfloor \mu_{0}\right)=\mu_{0} \mu_{1}$. This is consistent with the range $[0.0,11.5)$ of Example 1, because we are now ignoring the $-\varepsilon_{\max }$ term.
Example 5: For $\mu_{1}=4.4, \mu_{0}=3.6$, leading to $\sigma_{1}=0.8$, an accuracy of \pm 0.4 in the residues would guarantee a dynamic range of $[0.0,18.0)$, matching $[0.0,17.6)$ obtained in Example 2, to within the ignored $-\varepsilon_{\max }$ term.

We next consider an arbitrary precision $\pm \varepsilon_{\max }$ for the two residues. First, we note that if either of the moduli is an integral multiple of $\sigma_{1}$, then the dynamic range will be limited to that given in Theorem 1 , provided $\varepsilon_{\max } \leq \sigma_{1} / 2$. This is because the sloped lines will eventually wrap around, tracing the same path as that beginning at point 0 . This occurs in Fig. 1, for example, limiting the dynamic range to $[0,12.0)$. On the other hand, in Fig. 3 , neither $\mu_{1}$ nor $\mu_{0}$ is divisible by $\sigma_{1}=0.8$, allowing the dynamic range to extend beyond $[0,18.0)$, provided $\varepsilon_{\max }<0.2$. In general, such an extension is possible if the residues of $\mu_{1}$ and $\mu_{0} \bmod \sigma_{1}$ are at least $2 \varepsilon_{\max }$

Theorem 2: Let $\sigma_{-1}=\mu_{1}, \sigma_{0}=\mu_{0}$, and, for $0 \leq i \leq j, \sigma_{i+1}$ $=\min \left(\left|\sigma_{i-1}\right|_{\sigma_{i}}, \sigma_{i}-\left|\sigma_{i-1}\right|_{\sigma_{i}}\right)$, where $j$ is the largest possible index for which $\sigma_{j} \geq 2 \varepsilon_{\max }$. If the residues $\rho_{1}$ and $\rho_{0}$ are accurate to within $\pm \varepsilon_{\max }$, then the dynamic range $\mu$ of CD-RNS is lower-bounded by:

$$
\mu_{0}\left(1+\left\lfloor\mu_{1} / \mu_{0}\right\rfloor\left\lfloor\mu_{0} / \sigma_{1}\right\rfloor\right)\left\lfloor\sigma_{1} / \sigma_{2}\right\rfloor\left\lfloor\sigma_{2} / \sigma_{3}\right\rfloor \cdots\left\lfloor\sigma_{j-1} / \sigma_{j}\right\rfloor
$$

Given that the expression above is rather complicated, an intuitive explanation is called for. If we remove the floor operators (which essentially model the waste due to unequal line spacings, of the types seen in Fig. 3) and assume $\sigma_{j}=\gamma=2 \varepsilon_{\max }$, both bounds reduce to $\mu_{0} \mu_{1} /\left(2 \varepsilon_{\max }\right)$, indicating that under ideal conditions, the supported dynamic range is directly proportional to the two moduli and varies inversely with the representation error bound. One implication of Theorem 2 is that the same $\left\{\mu_{1}, \mu_{0}\right\}$ pair of moduli can be used to cover a wider dynamic range with more accurate residues, provided certain conditions on the parameters $\sigma_{i}$ are met.

Theorem 3: Define $\gamma=\max \left(2 \varepsilon_{\max }, \delta\right)$, where $\delta$ is the largest number that exactly divides both $\mu_{0}$ and $\mu_{1}$, if such a number exists, and 0 otherwise. Then, the dynamic range $\mu$ of the CD-RNS is upper-bounded by:

$$
\max \left(\mu_{0}\left\lfloor\mu_{1} / \gamma\right\rfloor, \mu_{1}\left\lfloor\mu_{0} / \gamma\right\rfloor\right)
$$

The lower/upper bounds defined in Theorems 2 and 3 are often close to each other, so that they provide a fairly accurate estimate for the exact dynamic range $\mu$. We will see shortly that optimal choice of the moduli essentially entails an attempt to render the lower and upper bounds as close to each other as possible. A useful variation of the result of Theorem 3 is the following:

Corollary 1: To attain a dynamic range $\mu$ with a maximum residue error $\varepsilon_{\max }$, we must have $\mu_{0} \mu_{1} \geq 2 \mu \varepsilon_{\max }$.

Example 6: Taking $\varepsilon_{\max }=0.2$ in the CD-RNS of Fig. 3, we obtain $\sigma_{1}=0.8$ and $\sigma_{2}=0.4$, with $\sigma_{3}=0.0$ violating the requirement for the last $\sigma_{j}$ being no less than $2 \varepsilon_{\max }$. The lower- and upper-bound expressions of Theorems 2 
and 3 evaluate to 36.0 and 39.6, respectively, indicating a dynamic range of at least $[0.0,36.0)$. Note that the true range is $[0.0,39.6)$, with its top end shown at the upper right corner of Fig. 3, that is, the point where full wraparound from 39.6 to 0 occurs.

Example 7: Raising the accuracy of residues in Example 6 to $\varepsilon_{\max }=0.1$ won't increase the dynamic range, given the full wraparound at 39.6. The pessimistic lower-bound expression of Theorem 2 reflects this limitation, as its value remains at 36.0 for any $\varepsilon_{\max }<0.2$. On the other hand, the optimistic upper bound of Theorem 3 indicates the wider range $[0.0,79.2)$, which is clearly unattainable. Slightly different values for the moduli $\mu_{1}$ and $\mu_{0}$ could fix this problem, however.

Example 8: We consider a special case where $\sigma_{1}$ is not an integral multiple of $\sigma_{2}$. For the CD-RNS of Fig. 4, we have $\sigma_{-1}=\mu_{1}=6.5, \sigma_{0}=\mu_{0}=4.4, \sigma_{1}=\left|\mu_{1}\right|_{\mu_{0}}=2.1$, and $\sigma_{2}=\left|\mu_{0}\right|_{\sigma_{1}}=0.2$. Assuming $\varepsilon_{\max }=0.1$, Theorem 2 yields the dynamic range lower bound 132.0. The upper end of the derived range is less than the ideal value $\mu_{1} \mu_{0} /\left(2 \varepsilon_{\max }\right)=143.0$, because some of the potential dynamic range is lost to the unequal spacing between lines of slope 1 in Fig. 4 and some to the fact that the lower bound is generally less than the true value. Note, in particular, that the solid line just below the one originating at 0 in Fig. 4 has a horizontal and vertical separations of 0.3 , instead of 0.2 . The next line after wraparound (dashed in Fig. 4) will have horizontal and vertical separations of 0.1 , which is clearly not allowed with $\varepsilon_{\max }=0.1$. The dynamic range is 136.5 .

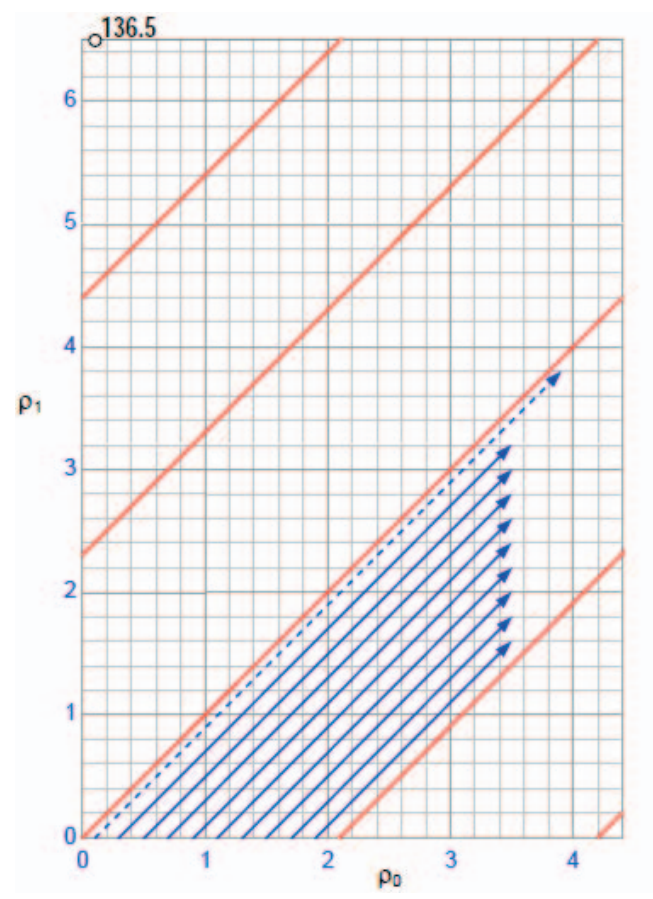

Fig. 4. Continuous-digit RNS with the moduli $\{6.5,4.4\}$.
Example 9: Consider a smaller error bound $\varepsilon_{\max }=0.05$ in Fig. 4. The dashed line, and its (wrapped) successors, are now allowed, leading to increases in the lower/upper bounds provided by Theorems 2 and 3 (to 264.0 and 286.0, respectively); the actual range is [0,286.0).

In conventional RNS, the moduli being pairwise relatively prime leads to maximization of the dynamic range. The corresponding result for CD-RNS is:

Theorem 4: Let $\sigma_{-1}=\mu_{1}, \sigma_{0}=\mu_{0}$, and, for $0 \leq i \leq j$, $\sigma_{i+1}=\min \left(\left|\sigma_{i-1}\right|_{\sigma_{i}}, \sigma_{i}-\left|\sigma_{i-1}\right|_{\sigma_{i}}\right)$, where $j$ is the largest index for which $\sigma_{j} \geq 2 \varepsilon_{\max }$. The maximum possible dynamic range of $\mu_{0} \mu_{1} /\left(2 \varepsilon_{\max }\right)$ is attained if $\sigma_{j}=2 \varepsilon_{\max }$.

Note that the maximum range specified by Theorem 4 is achieved in Examples 1, 3, 4, 6, and 9.

\section{Choosing the CD-RNS Moduli}

Based on Theorem 1, given a maximum error $\pm \varepsilon_{\max }$ in residues, we can choose the moduli as $\mu_{0}+2 \varepsilon_{\max }$ and $\mu_{0}$, leading to the dynamic range $\mu_{0}\left\lfloor\mu_{0} /\left(2 \varepsilon_{\max }\right)\right\rfloor+\mu_{0}$. This observation suggests that to cover a desired dynamic range $[0, \mu)$ with residues that are accurate to $\pm \varepsilon_{\max }$ and two moduli that are as small as possible, $\mu_{1}$ and $\mu_{0}$ should roughly equal $\left(2 \mu \varepsilon_{\max }\right)^{1 / 2}$ and they should differ by $2 \varepsilon_{\max }$.

Example 10: Dynamic range $[0,100.0)$ with $\varepsilon_{\max }=0.1$ translates to moduli of the order of 4.5. Choosing $\mu_{1}=4.6$ and $\mu_{0}=4.4$ yields the dynamic range $[0.0,101.2)$.

Note that the dynamic range of Example 10 could have been computed by scaling up $\varepsilon_{\max }$ to 1 , scaling up the two moduli by the same factor of 10 to integer values $m_{1}=46$ and $m_{0}=44$, and then using the rules for ordinary RNS. This would have yielded $M=m_{1} m_{0} / \operatorname{gcd}\left(m_{1}, m_{0}\right)=1012$. Scaling back down by a factor of 10 confirms the derived range of $[0.0,101.2)$. However, the same could not be done when 1 is not an integral multiple of $\varepsilon_{\max }$.

Example 11: For attaining the dynamic range defined by $\mu=100.0$, with $\varepsilon_{\max }=0.15$, the two moduli must be on the order of $(2 \times 100.0 \times 0.15)^{1 / 2}=5.5$. Choosing $\mu_{1}=5.6$ and $\mu_{0}=5.4$ yields the dynamic range $[0,102.6)$. There is no direct counterpart to this latter CD-RNS among ordinary residue number systems.

Now consider the case when a rough magnitude for one modulus is known. Without loss of generality, let the smaller of the two moduli $\mu_{0}$ be given approximately. Further, assume that the given $\mu_{0}$ is an integral multiple of $2 \varepsilon_{\max }$ (if not, minor adjustment to $\mu_{0}$ will make it so). Then, to achieve a dynamic range $[0, \mu)$, Corollary 1 suggests that $\mu_{1} \geq 2 \mu \varepsilon_{\max } / \mu_{0}$. One way to ensure that a near-minimal value for $\mu_{1}$ would be adequate is to adjust it upward, if necessary, until it is a multiple of $2 \varepsilon_{\max }$, compute the lower bound of Theorem 2, and continue adjusting upward by $2 \varepsilon_{\max }$ until the lower bound equals or exceeds the desired dynamic range. Alternatively, one could aim for satisfying the condition of Theorem 4 . 
Example 12: Consider a stated goal of $\mu \geq 100.0$, with $\varepsilon_{\max }=0.15$ and $\mu_{0} \approx 3.5$. We take $\mu_{0}=3.6$, which is an integral multiple of $2 \varepsilon_{\max }$. Corollary 1 yields $\mu_{1} \geq 8.33$. Taking $\mu_{1}=8.4$ leads to the lower bound of 25.2 , which is too small. Adding $=0.3$ to make $\mu_{1}=8.7$ yields the lower bound 72.0 , which is still inadequate. We could continue with increasing $\mu_{1}$ to 9.0 and beyond, but Theorem 4 guarantees a dynamic range of $[0,104.4)$, with $\mu_{0}=3.6$ and $\mu_{1}=8.7$, given that $\sigma_{3}=0.3$.

\section{An Application of CD-RNS}

This study of continuous-digit RNS and their properties was motivated by the following problem in computational neurobiology. It is postulated [13] that the common rat's uncanny ability to return to its starting position in a straight line, after a long sequence of movements in different directions, in the absence of any visual markers (or even in total darkness), results from internal neural activity as opposed to the processing of environmental clues. Briefly, a landmark paper [14] established that in addition to "place cells," activated when the rat is in specific locations, there exist grid cells whose firing is not linked to specific locations, but rather to the rat's relative in-cell position within a periodic, hexagonal grid.

For simplicity, let us ignore the 2D (or even 3D) nature of a rat's environs and focus on a 1D model. As shown in Fig. 5, given a 1D grid $x$, the real value $R$ might be specified by a pair $(i, \phi)$, where $i$ is an integer identifier of a grid cell or interval and $\phi$ is a real-valued "phase" or displacement within the cell. Similarly, $R$ can be specified by the pair $(j, \psi)$ in relation to a second $1 \mathrm{D}$ grid $y$. Now, given only the phases $\phi$ (associated with any of the heavy dots in Fig. 5) and $\psi$ (hollow circles) within the grids $x$ and $y$, we may be able to deduce $R$.

There are several open problems in computational neurobiology with regard to the rat's navigation system. One such problem is the dynamic range of the aforementioned location representation scheme with a given set of moduli. Numerical simulations indicate that the range is an exponentially increasing function of $1 / \varepsilon_{\max }$, with the exponent being slightly less than the number of moduli. For example, an exponent of 10.7 is observed with a particular set of 12 moduli [13]. Applying our results to the same case, we obtain an exponent of 11.0, which is in good agreement with experiments.

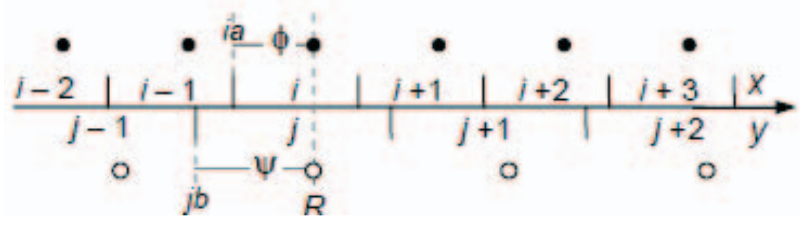

Fig. 5. Localization with two grids in 1D space.

\section{Conclusions}

Our results provide a digital arithmetic perspective on the work of computational neuroscientists who have hypothesized the use of modular number representation of location data in rats (and other animals) and have verified the feasibility of this type of representation, as well as adequacy of the resulting dynamic range via simulation. Our theoretical results on CD-RNS confirm the feasibility of these hypotheses.

Research can continue in a number of directions. In the domain of digital arithmetic, one can complete and expand the theory presented here by removing some restrictions. In the realm of biology, one may try to determine how close the actual moduli associated with a rat's navigation system are to optimal values and whether there is any evidence of evolutionary refinement of the moduli or the representational accuracy.

\section{References}

[1] Garner, H. L., "The Residue Number System," IEEE Trans. Electronic Computers, Vol. 8, pp. 140-147, 1959.

[2] Svoboda, A., "The Numerical System of Residual Classes in Mathematical Machines," in Information Processing (Proc. UNESCO Conf., June 1959), pp. 419-422, 1960.

[3] Omondi, A., and B. Premkumar, Residue Number Systems: Theory and Implementation, Imperial College Press, 2007.

[4] Parhami, B., Computer Arithmetic: Algorithms and Hardware Designs, Oxford Univ. Press, 2nd ed., 2010.

[5] Soderstrand, M. A., W. K. Jenkins, G. A. Jullien, and F. J. Taylor (eds.), Residue Number System Arithmetic, IEEE Press, 1986.

[6] Bernocchi, G. L., G. C. Cardarilli, A. Del Re, A. Nannarelli, and M. Re, "Low-Power Adaptive Filter Based on RNS Components," Proc. Int'l Symp. Circuits and Systems, pp. 3211-3214, 2007.

[7] Barsi, F., and P. Maestrini, "Error Correcting Properties of Redundant Residue Number Systems," IEEE Trans. Computers, Vol. 22, No. 3, pp. 307-315, 1973.

[8] Saed, A., M. Ahmadi, and G. A. Jullien, "Arithmetic with Signed Analog Digits," Proc. 14th IEEE Symp. Computer Arithmetic, pp. 134-141, 1999.

[9] Saed, A., M. Ahmadi, and G. A. Jullien, "A Number System with Continuous Valued Digits and Modulo Arithmetic," IEEE Trans. Computers, Vol. 51, No. 11, pp. 1294-1305, 2002.

[10] Mirhassani, M., M. Ahmadi, and G. A. Jullien, "LowPower Mixed-Signal CVNS-Based 64-Bit Adder for Media Signal Processing," IEEE Trans. VLSI Systems, Vol. 16, No. 9, pp. 1141-1150, 2008.

[11] Kim, J. Y., K. H. Park, and H. S. Lee, "Efficient Residueto-Binary Conversion Technique with Rounding Error Compensation," IEEE Trans. Circuits and Systems, Vol. 38, pp. 315-317, 1991.

[12] Hung, C. Y. and B. Parhami, "Error Analysis of Approximate Chinese-Remainder-Theorem Decoding," IEEE Trans. Computers, Vol. 44, No. 11, pp. 1344-1348, 1995.

[13] Fiete, I. R., Y. Burak, and T. Brookings, "What Grid Cells Convey about Rat Location," J. Neuroscience, Vol. 28, No. 27, pp. 6858-6871, 2008.

[14] Hafting, T., M. Fyhn, S. Molden, M.-B. Moser, and E. I. Moser, "Microstructure of a Spatial Map in the Entorhinal Cortex," Nature, Vol. 436, pp. 801-806, 2005. 